\title{
SCIENTIFIC REPORTS

\section{High-fat diet-mediated dysbiosis exacerbates NSAID-induced small intestinal damage through the induction of interleukin-17A}

\begin{abstract}
Naoki Sugimura ${ }^{1}$, Koji Otani ${ }^{1}{ }^{1}$, Toshio Watanabe ${ }^{1 *}$, Geicho Nakatsu $^{4}$, Sunao Shimada ${ }^{1}$, Kosuke Fujimoto ${ }^{2,3}$, Yuji Nadatani ${ }^{1}$, Shuhei Hosomi ${ }^{1}$, Fumio Tanaka ${ }^{1}$, Noriko Kamata ${ }^{1}$, Koichi Taira ${ }^{1}$, Yasuaki Nagami ${ }^{1}$, Tetsuya Tanigawa ${ }^{1}$, Satoshi Uematsu ${ }^{2,3}$ \& Yasuhiro Fujiwara ${ }^{1}$

Non-steroidal anti-inflammatory drugs (NSAIDs) cause damage in the small intestine in a bacteriadependent manner. As high-fat diet (HFD) is a potent inducer of gut dysbiosis, we investigated the effects of HFD on bacterial flora in the small intestine and NSAID-induced enteropathy. 16S rRNA gene analysis revealed that the population of Bifidobacterium spp. significantly decreased by fold change of individual operational taxonomic units in the small intestine of mice fed HFD for 8 weeks. HFD increased intestinal permeability, as indicated by fluorescein isothiocyanate-dextran absorption and serum lipopolysaccharide levels, accompanied by a decrease in the protein expressions of ZO-1 and occludin and elevated mRNA expression of interleukin (IL)-17A in the small intestine. HFD-fed mice exhibited increased susceptibility to indomethacin-induced damage in the small intestine; this phenotype was observed in normal diet-fed mice that received small intestinal microbiota from HFDfed mice. Administration of neutralizing antibodies against IL-17A to HFD-fed mice reduced intestinal permeability and prevented exacerbation of indomethacin-induced damage. Thus, HFD-induced microbial dysbiosis in small intestine caused microinflammation through the induction of IL-17A and increase in intestinal permeability, resulting in the aggravation of NSAID-induced small intestinal damage.
\end{abstract}

Non-steroidal anti-inflammatory drugs (NSAIDs) are the most widely used class of drugs for the treatment of inflammatory conditions and pain management. It is well known that the use of NSAIDs is associated with gastrointestinal damage in aging populations. In addition, several studies using video capsule endoscopy (CE) have demonstrated that NSAIDs can damage the small intestine as well as the upper gastrointestinal tract ${ }^{1-3}$. Although the adverse effects of NSAIDs on the small intestine have been recognized as a healthcare crisis, the understanding of the factors that potentiate NSAID-induced small intestinal damage is limited. Gastric acid is not involved in NSAID-induced small intestinal damage; hence, acid secretion inhibitors, such as proton pump inhibitors (PPIs), cannot provide therapeutic effects against the damage. On the contrary, Wallace et al. reported that PPIs alter the composition of rat intestinal bacteria, including the reduction of jejunal Actinobacteria and Bifidobacteria spp., which results in the exacerbation of NSAID-induced small intestinal ulcers ${ }^{4}$. Together with the facts that intestinal bacteria play an important role in the pathophysiology of small intestinal damage by their capacity to activate the innate immune system, this microbiome study also offers the possibility that factors affecting the small intestinal flora could aggravate NSAID-induced small intestinal damage.

Abnormality in the composition of the microbial community is known as dysbiosis, and this condition is associated with various diseases, e.g., inflammatory bowel disease ${ }^{5}$, non-alcoholic fatty liver disease ${ }^{6}$, and colorectal

${ }^{1}$ Department of Gastroenterology, Osaka City University Graduate School of Medicine, 1-4-3 Asahimachi, Abeno-ku, Osaka, 545-8585, Japan. '2Department of Immunology and Genomics, Osaka City University Graduate School of Medicine, 1-4-3 Asahimachi, Abeno-ku, Osaka, 545-8585, Japan. ${ }^{3}$ Division of Innate Immune Regulation, International Research and Development Center for Mucosal Vaccines, The Institute of Medical Science, The University of Tokyo, 4-6-1 Shirokanedai, Minato-ku, Tokyo, 108-8639, Japan. ${ }^{4}$ Department of Immunology and Infectious Diseases/Genetics and Complex Diseases, Harvard T. H. Chan School of Public Health, Room 904, Building 1, 665 Huntington Avenue, Boston, Massachusetts, 02115, United States. *email: watanabet@med.osaka-cu.ac.jp 
A

\section{Control}

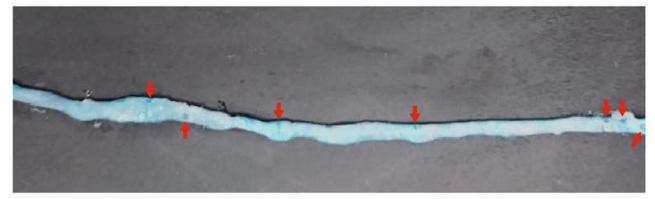

HFD

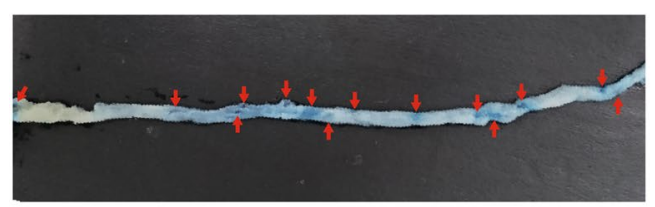

C

Control

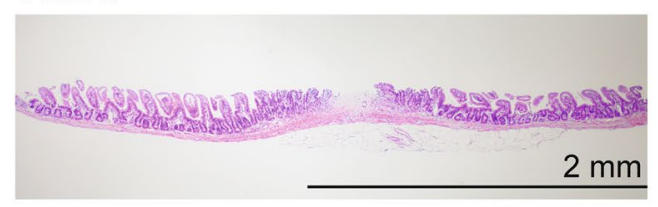

HFD

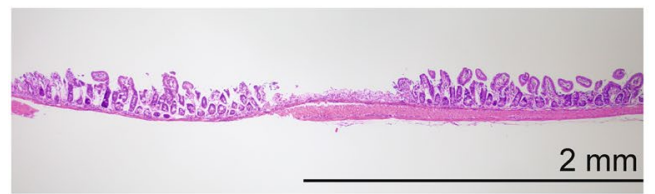

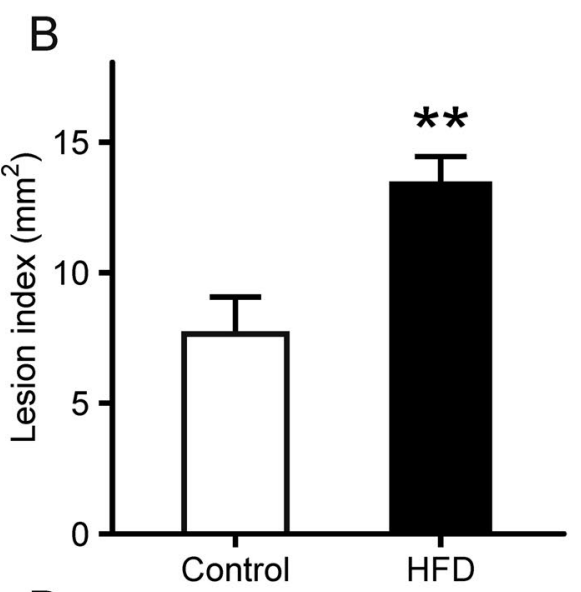

D

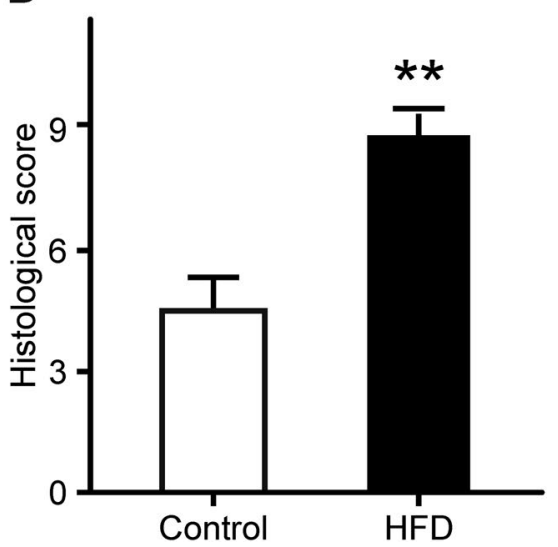

Figure 1. Small intestinal damage after indomethacin administration in HFD-fed mice. (A) Damaged mucosa was stained dark blue with 1\% Evans blue (arrows). (B) Lesion index after administration of indomethacin. The area $\left(\mathrm{mm}^{2}\right)$ of macroscopically visible lesion was measured, summed per small intestine, and designated as the lesion index. $n=7-9$. (C) Representative histological images of indomethacin-induced small intestinal damage (hematoxylin and eosin stain). (D) Histological evaluation of indomethacin-induced small intestinal damage. $\mathrm{n}=4-5$. Each column represents mean \pm standard error of the mean. $* * p<0.01$ vs. control mice. Control, control group fed AIN-93M; HFD, HFD group fed HFD-60.

cancer ${ }^{7}$. In addition, dysbiosis is closely related to dietary factors, and high-fat diet (HFD) is a potent inducer of dysbiosis which can result in the promotion of obesity ${ }^{8}$. HFD-induced dysbiosis attenuates intestinal barrier function $^{9}$, resulting in leaky gut that causes metabolic endotoxemia, inflammation, and associated disorders due to increased intestinal permeability ${ }^{10}$. Therefore, it is important to accurately evaluate the dietary factors to prevent the NSAID-induced small intestinal damage.

In this study, we investigated changes in bacterial flora and permeability within the small intestine after HFD intake and their influence on NSAID-induced small intestinal damage in mice.

\section{Results}

Exacerbation of indomethacin-induced small intestinal damage in mice fed HFD. Mice were randomly separated into a control group fed AIN-93M (normal diet) or an HFD group fed HFD-60 for 8 weeks. Body weight (BW) was recorded weekly, and BW in the HFD group significantly increased compared with that of the control group (Supplementary Fig. S1). Mice were subsequently administered indomethacin ( $12 \mathrm{mg} / \mathrm{kg})$ by gavage and sacrificed $24 \mathrm{~h}$ later. Lesion index, the summed area $\left(\mathrm{mm}^{2}\right)$ of macroscopically visible lesion as observed with $1 \%$ Evans blue staining, increased significantly by 2.0 -fold in the HFD group, compared with that in the control group $\left(p=6.4 \times 10^{-3}\right.$; Fig. $\left.1 \mathrm{~A}, \mathrm{~B}\right)$. In addition, histological examination of samples from the HFD group showed intestinal sloughing and destruction of the upper epithelium with infiltration of inflammatory cells as well as mucosal ulceration, which extended into the submucosal layer; these observations suggest relatively severe indomethacin-induced mucosal damage $\left(p=2.5 \times 10^{-3}\right.$; Fig. $\left.1 \mathrm{C}, \mathrm{D}\right)$.

HFD-induced alterations in the small intestinal microbiota. We performed subsequent experiments using HFD-treated small intestine prior to indomethacin administration. To investigate HFD-associated dysbiosis in the small intestine, the V3-V4 components of luminal microbiome samples were amplified and subjected to $16 \mathrm{~S}$ rRNA gene sequence analysis. Comparative analysis of bacterial $\alpha$-diversity metrics showed a reduced richness of operational taxonomic units (OTUs) in the class Actinobacteria $\left(p=6.3 \times 10^{-4}\right.$, false 
discovery rate $\left.[\mathrm{FDR}]=1.8 \times 10^{-2}\right)$, the families Bifidobacteriaceae $\left(p=3.1 \times 10^{-4}, \mathrm{FDR}=1.3 \times 10^{-2}\right)$, and Streptococcaceae $\left(p=1.2 \times 10^{-3}, \mathrm{FDR}=2.3 \times 10^{-2}\right)$, and increased OTU diversity in the order Clostridiales $\left(p=4.2 \times 10^{-3}, \mathrm{FDR}=5.9 \times 10^{-2}\right)$, and the families Erysipelotrichaceae $\left(p=1.1 \times 10^{-7}, \mathrm{FDR}=2.9 \times 10^{-6}\right)$, and Ruminococcaceae $\left(p=1.3 \times 10^{-4}, \mathrm{FDR}=2.7 \times 10^{-3}\right)$ in the HFD group (Fig. 2A). The overall richness and diversity of microbiota OTUs were not significantly different (Supplementary Fig. S2). Principal coordinate analysis (PCoA) of Bray-Curtis distances among the OTU profiles showed a clear separation between the HFD group and the control group (PERMANOVA, $p=1.0 \times 10^{-3}$; Fig. $2 \mathrm{~B}$ ). Fold change analysis of individual OTUs suggested that the population of Bifidobacterium spp. decreased significantly in the HFD group $\left(p=1.0 \times 10^{-6}\right.$, $\mathrm{FDR}=8.1 \times 10^{-5}$; Fig. $2 \mathrm{C}$ ). Heatmap visualization further demonstrated little sample variances in the depletion and enrichment of the bacterial OTU components across the dietary groups, which were independent of cage effects (Fig. 2D).

Increase in intestinal permeability in HFD-fed mice. Small intestinal permeability was examined by measuring fluorescein isothiocyanate (FITC)-dextran and serum lipopolysaccharide (LPS) that is a membrane component found in gram-negative bacteria. Serum FITC-dextran in the HFD group was significantly elevated by 2.0 -fold prior to indomethacin administration compared with that in the control group $\left(p=3.3 \times 10^{-4} ;\right.$ Fig. $\left.3 \mathrm{~A}\right)$. This result was consistent with changes in serum LPS levels $\left(p=5.2 \times 10^{-3}\right.$; Fig. 3B).

Effects of HFD treatment on the expressions of inflammatory cytokines and tight junction components. We measured the expressions of inflammatory cytokines, innate immune receptors, and tight junction components in the small intestine of HFD-fed mice prior to indomethacin administration. The mRNA levels of interleukin (IL)-17A (Fig. 4A) and MCP-1 (Fig. 4B) in the HFD group were significantly increased by 3.5 -fold $\left(p=7.8 \times 10^{-3}\right)$ and 1.5 -fold $\left(p=3.3 \times 10^{-3}\right)$, respectively, although there were no differences in the expressions of other inflammatory cytokines, such as IL-1 $\beta$, TNF- $\alpha$, and toll-like receptor (TLR) 4 (Fig. 4C-E). The mRNA levels of TLR 9 decreased significantly in the HFD group $\left(p=3.8 \times 10^{-2}\right.$; Fig. 4F), and the expressions of tight junction proteins, such as ZO-1 (Fig. $4 \mathrm{H}$ ) and occludin (Fig. 4I), were reduced in the small intestinal mucosa in the HFD group.

Effects of neutralizing IL-17A on indomethacin-induced small intestinal damage in HFD-fed mice. To determine the role of IL-17A, we administered anti-IL-17A antibody or isotype control to HFD-fed mice prior to indomethacin treatment once daily for 7 days. Two days after the final dose, we administered indomethacin $(12 \mathrm{mg} / \mathrm{kg} \mathrm{BW})$ by gavage, and the mice were sacrificed $24 \mathrm{~h}$ later. Lesion index was significantly reduced in the HFD group treated with anti-IL-17A antibodies $\left(p=9.3 \times 10^{-3}\right.$; Fig. 5A), and this result was consistent with the histological observations $\left(p=8.0 \times 10^{-4}\right.$; Fig. 5B). Furthermore, IL-17A neutralization significantly reduced intestinal permeability and serum LPS in the HFD group $\left(p=1.4 \times 10^{-2}\right.$; Fig. 5 C; $p=3.5 \times 10^{-2}$; Fig. 5D).

Transplantation of altered small intestinal microbiota from HFD-fed mice. To investigate whether microbiota modulates indomethacin-induced small intestinal damage, small intestinal luminal contents collected from donor mice fed HFD-60 or AIN-93M were transplanted into microbiota-depleted recipient mice, treated with antibiotic cocktail, fed AIN-93M once daily for 5 days. One day after the final transplantation, mice were administered indomethacin $(12 \mathrm{mg} / \mathrm{kg} \mathrm{BW})$ by gavage and sacrificed $24 \mathrm{~h}$ later (Fig. $6 \mathrm{~A})$. As a result, intestinal microbiota transplantation from HFD-60-fed mice exacerbated small intestinal damage, and significantly increased lesion index $\left(p=1.1 \times 10^{-2}\right.$; Fig. 6B) and histological score $\left(p=1.1 \times 10^{-3}\right.$; Fig. 6C) were observed.

\section{Discussion}

In this study, we demonstrated that HFD treatment changed the gut microbiota in the small intestine and exacerbated NSAID-induced small intestinal damage. As the small intestinal microbiota plays an underlying role in the regulation of endotoxemia and inflammatory response, it is essential to identify major bacteria present in the small intestine of HFD-treated mice. Microbiota analysis showed that the proportion of gram-positive anaerobic bacilli, Bifidobacterium spp., was significantly reduced in HFD-fed mice. It has been reported that PPI-treatment exacerbated NSAID-induced small intestinal injury by inducing the reduction of Bifidobacteria spp., and recolonization with Bifidobacteria-enriched commensal bacteria prevented the damage ${ }^{4}$. Bifidobacterium spp. do not degrade intestinal mucus glycoproteins, but, instead, promote a stable environment and improve gut barrier function by inhibiting the translocation of pathogenic enterobacteria and endotoxin ${ }^{11,12}$. By contrast, increasing the proportion of gram-negative bacteria can decrease the integrity of intestinal mucosa and lead to metabolic endotoxemia $^{13}$. In this study, it is assumed that a decrease in Bifidobacterium spp. was involved in intestinal hyperpermeability and metabolic endotoxemia observed in HFD-fed mice.

Intestinal permeability is regulated by the components of the epithelial cellular tight junction, such as ZO-1 and occludin. In this study, the protein expressions of ZO-1 and occludin decreased in the small intestinal mucosa of HFD-fed mice. The dysfunction of intestinal tight junction barrier is associated with intestinal microbiota dysbiosis ${ }^{14}$. Hence, we proposed that the disruption of intestinal tight junction components in this study was accelerated by HFD-induced dysbiosis, which exacerbated intestinal permeability. Consequently, endotoxin was increased in the blood, triggering a cascade of inflammatory responses. In a previous study, the administration of NSAID accelerated intestinal permeability during absorption ${ }^{15}$ and resulted in the induction of mucosal damage. In the present study, we assume that HFD-induced intestinal barrier dysfunctions facilitated enterobacterial invasion of mucosae and metabolic endotoxemia, which may have promoted the development of indomethacin-induced small intestinal damage.

We previously reported that when mucosal barrier function is disrupted, LPS and high-mobility group box 1 from the injured epithelial cells bind to TLR 4 and activate NF- $\mathrm{B}$, through the MyD88-dependent pathway 


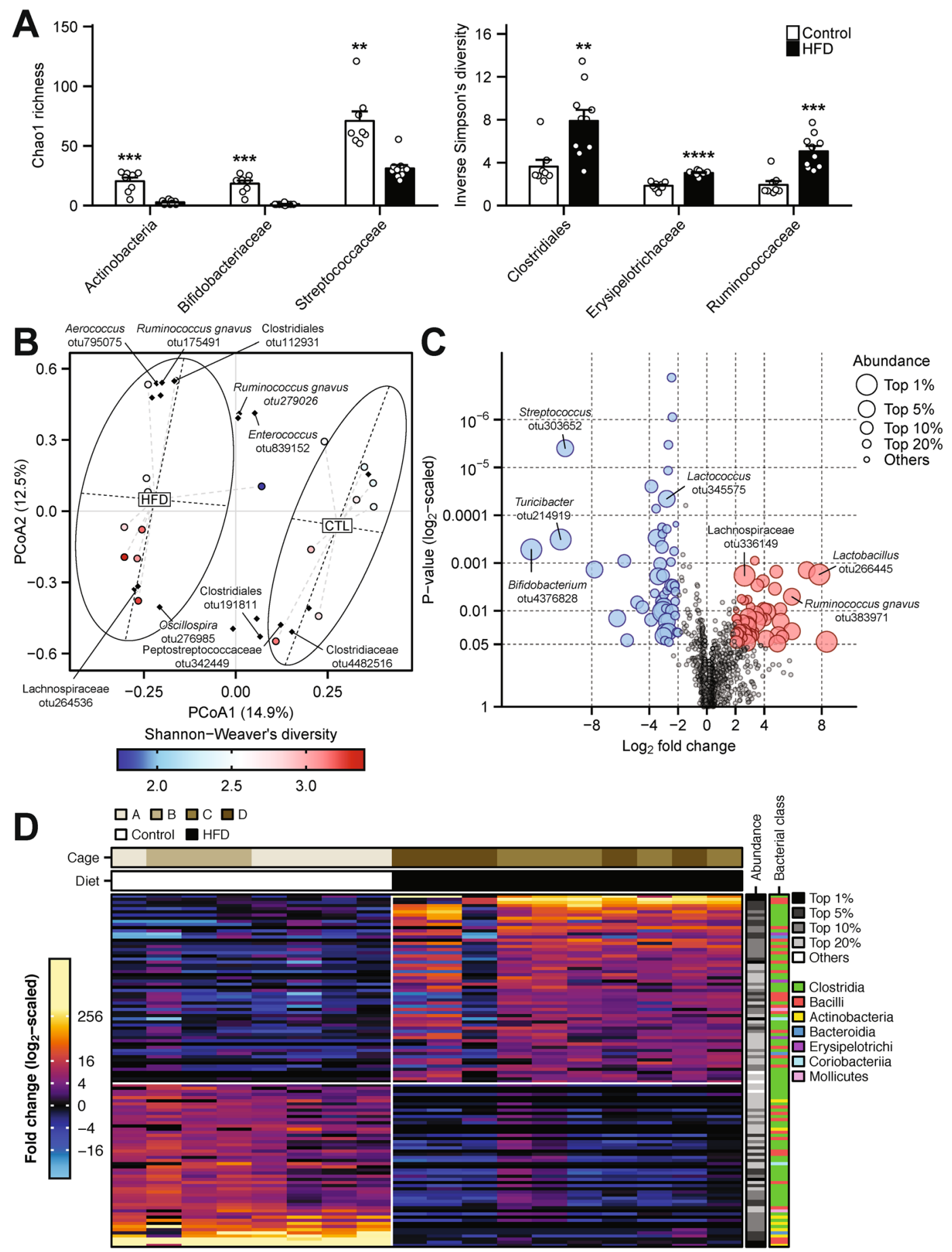

Figure 2. $16 \mathrm{~S}$ rRNA gene analysis of small intestinal microbiome. (A) $\alpha$-diversity analysis of luminal microbiota operational taxonomic unit (OTU) at various taxonomic ranks. $p$-values are from two-tailed Student's t-tests. (B) Principal coordinate analysis (PCoA) of $\beta$-diversity based on Bray-Curtis dissimilarity matrix of OTU-level compositional profiles. Ellipses represent $95 \%$ confidence intervals. Solid diamondshaped points in black denote species scores, which were calculated using the vegan R-CRAN package. (C) Volcano plot showing relative trimmed-mean robust fold change in OTU abundance compared with the control group. $P$-values are computed by fitting multiple linear models with generalized least squares and derived by empirical Bayesian moderation of $\mathrm{t}$-statistics on the fitted models as implemented in limma R/Bioconductor package. (D) Heatmap analysis of significantly enriched and depleted bacterial OTUs. $* * p<0.01, * * * p<0.001$, $* * * * p<0.0001$ vs. control mice. Control, control group fed AIN-93M; HFD, HFD group fed HFD-60.

and the NLR family pyrin domain-containing 3 inflammasome. This leads to the release of proinflammatory cytokines, such as TNF- $\alpha$ and IL-1 $\beta$, in NSAID-induced small intestinal damage ${ }^{16-18}$. Although the mRNA levels of TLR4, TNF- $\alpha$, and IL- $1 \beta$ did not change, the mRNA level of TLR9 decreased in small intestine treated with 
A

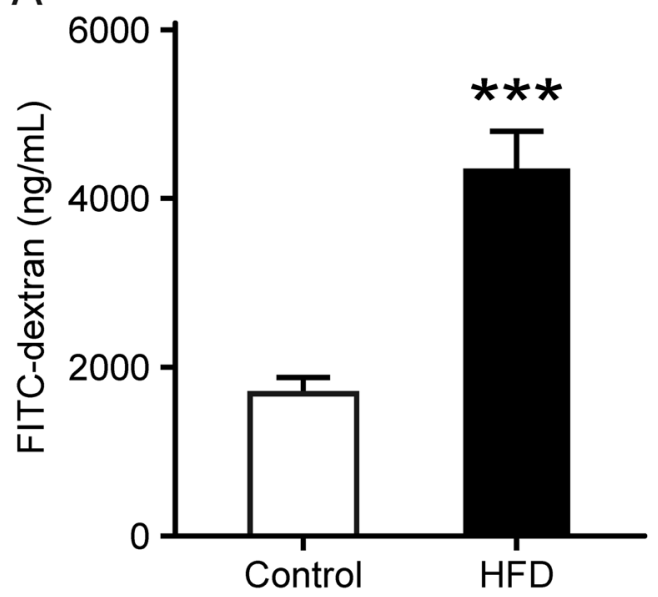

B

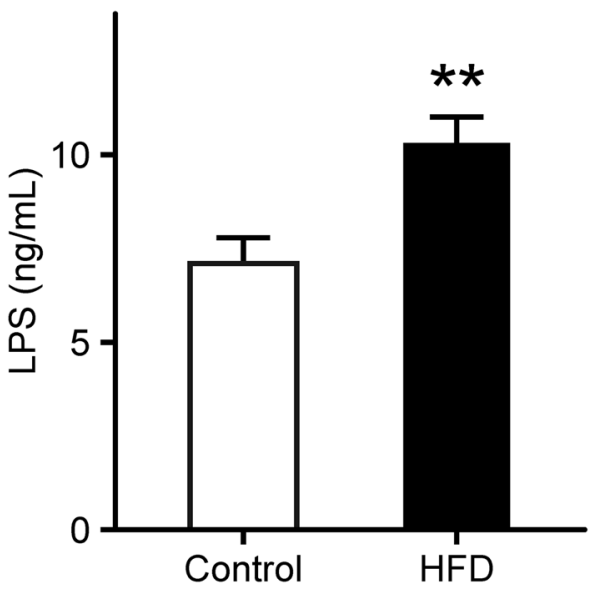

Figure 3. Gut leakiness in HFD-fed mice. (A) Intestinal permeability was examined by measuring serum FITC-dextran levels. $\mathrm{n}=10$. (B) Serum LPS levels were analyzed using the murine LPS ELISA kit. $\mathrm{n}=8$. Each column represents mean \pm standard error of the mean. ${ }^{*} p<0.01, * * * p<0.001$ vs. control mice. Control, control group fed AIN-93M; HFD, HFD group fed HFD-60.

HFD before NSAID administration. TLR9 is involved in the activities of macrophages against bacterial infection ${ }^{19}$ by identifying unmethylated CpG-DNA sites in bacterial $\mathrm{DNA}^{20}$. It is considered a protective factor in intestinal homeostasis, and Western diets are associated with its dysfunction ${ }^{21}$. Long-term administration of HFD in this study may have reduced the expression of TLR9 resulting in the deactivation of its protective function in this study.

We demonstrated that IL-17A was elevated in HFD-treated small intestine, and the neutralization of IL-17A improved the indomethacin-induced damage, intestinal permeability, and characteristics of endotoxemia. IL-17A is a pro-inflammatory cytokine associated with a variety of inflammatory diseases ${ }^{22}$, and there are several reports indicating that HFD intake induces the expression of IL-17 and exacerbates inflammatory diseases, e.g., psoriasis, colitis, and steatohepatitis ${ }^{23-25}$. Recent studies suggested that HFD-induced microbial alterations, especially the expansion of Firmicutes ${ }^{26,27}$, might be responsible for IL-17 induction. It is possible that the increased populations of Clostridiales and Erysipelotrichaceae belonging to Firmicutes in the HFD group might have enhanced the expression of IL-17A, thereby promoting small intestinal microinflammation and intestinal permeability in this study. Our result aligns with that of a previous study, which demonstrated that gut epithelial barrier dysfunctions can be reversed by IL-17A neutralization in a neuro-psychoactive drug-induced bacterial infection model ${ }^{28}$. Therefore, a therapeutic approach targeting IL-17A may help to diminish the metabolic burden of endotoxemia caused by defective intestinal barrier functions. A deeper mechanistic understanding of how IL-17A may interact with microbiota for homeostatic control of intestinal epithelia is warranted for the development of a novel therapeutic strategy against NSAID-induced small intestinal damage.

In previous studies, it has been demonstrated that intestinal microbiota is involved in the pathogenesis of NSAID-induced enteropathy ${ }^{29-31}$. Therefore, drugs regulating intestinal flora, such as probiotics, can be utilized as therapeutic agents ${ }^{32}$. It is known that Clostridium difficile-induced colitis can be remarkably restored by changing intestinal flora through feces transplantation from healthy subjects into the large intestine of patients; hence, fecal microbiota transplantation (FMT) can be applied to various other intestinal diseases ${ }^{33}$. There is a scarcity of FMT studies on the flora of small intestine as most FMT studies have examined the bacterial flora of large intestine. In this study, small intestinal microbial transplantation from HFD-fed mice exacerbated indomethacin-induced small intestinal damage. The alterations of small intestinal microbiota with HFD treatment may have increased the susceptibility of mucosae and they were sufficient to influence on indomethacin-induced enteropathy. To the best of our knowledge, this is the first report demonstrating that HFD-induced dysbiosis forms the substrate to aggravate indomethacin-induced enteropathy through IL-17A.

In conclusion, HFD-induced alterations of small intestinal microbiota cause microinflammation through the induction of IL-17A, and an increase in intestinal permeability, which aggravate NSAID-induced small intestinal damage. Low-fat dietary therapy and strategies to enhance individual-specific innate functions of Bifidobacterium may be useful for the prevention and mitigation of NSAID-induced small intestinal damage. A therapeutic strategy targeting IL-17A could be a treatment option for such enteropathy.

\section{Methods}

Animals and diets. Specific pathogen-free male C57BL/6 J mice (4 weeks old, 10-15g) were purchased from Charles River Japan Inc. (Atsugi, Japan). Animals were housed in polycarbonate cages with paper-chip bedding in an air-conditioned biohazard room with a 12-h light/dark cycle. All animals had free access to food and water. Mice were acclimated for 1 week prior to the experiments and received regular chow (CE-2, CLEA Japan Inc., Tokyo, Japan). At 6 weeks of age, mice were randomly separated into a control group fed AIN-93M (Oriental 

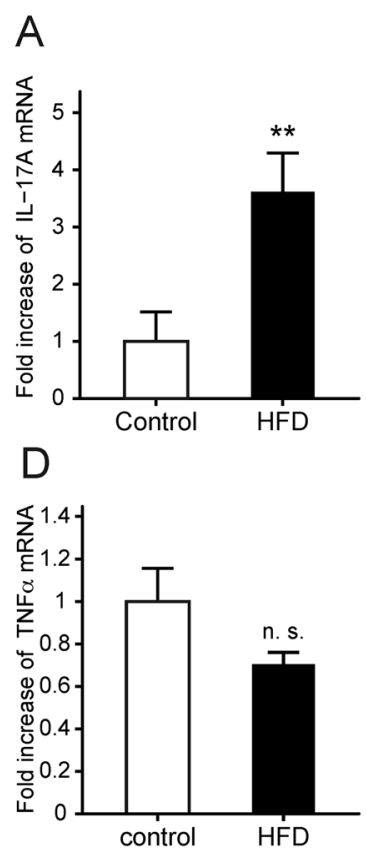

G

B

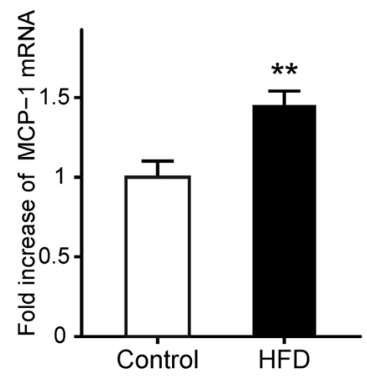

E

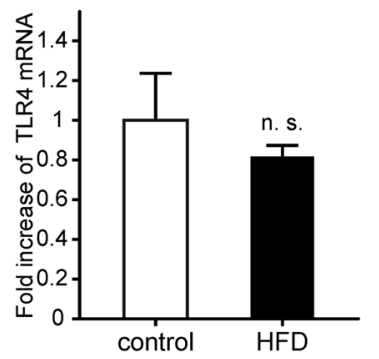

C

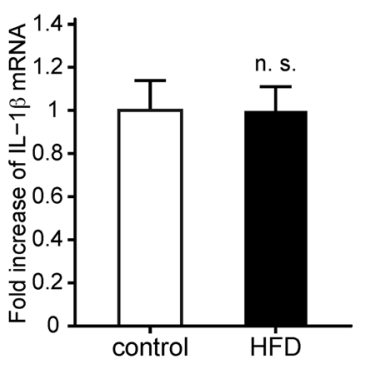

$\mathrm{F}$

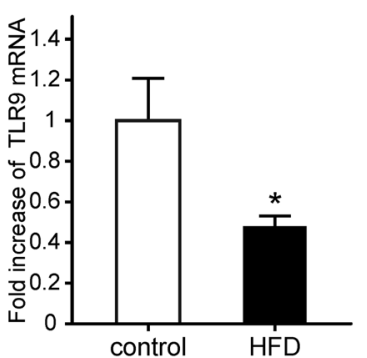

\section{Occludin}

Control

HFD

$\beta$-actin

$42 \mathrm{kDa}$

$\mathrm{H}$

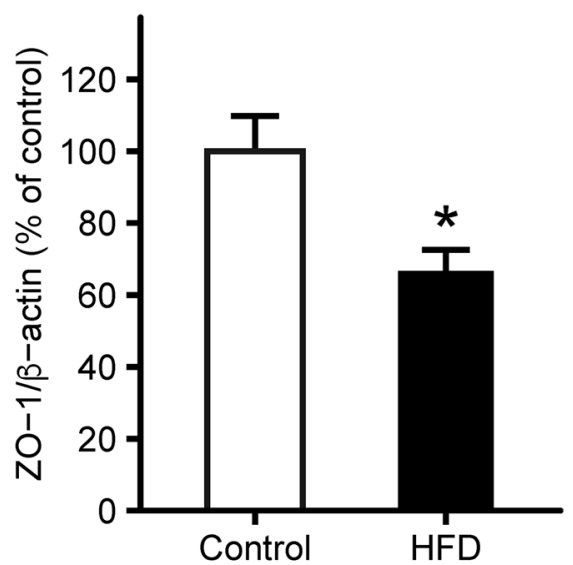

I

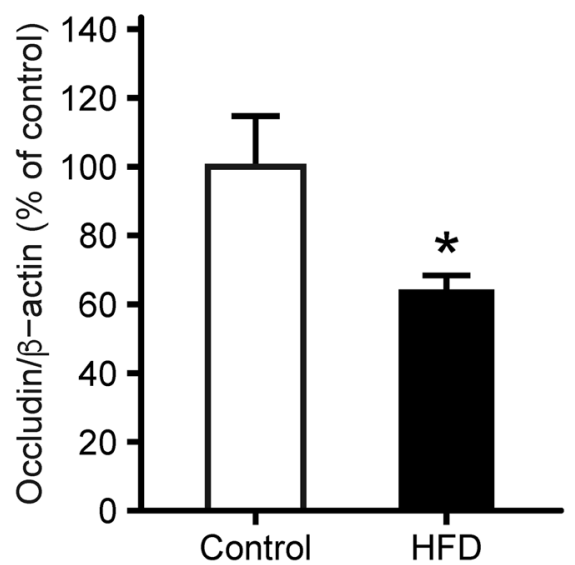

Figure 4. Small intestinal expressions of cytokines and tight junction components in HFD-fed mice. (A-F) Expressions of IL-17A (A), MCP-1 (B), IL-1 $\beta$ (C), TNF- $\alpha$ (D), TLR4 (E), and TLR9 (F) were evaluated by real-time quantitative RT-PCR. The mRNA levels are expressed as ratios of the mean value for control mice. $\mathrm{n}=9-10$. (G) Representative images of western blots for ZO-1 and occludin. Quantitative analyses of protein expressions for ZO-1 (H) and occludin (I) in the small intestine of HFD-fed mice. Protein levels are normalized to that of $\beta$-actin. $\mathrm{n}=6-7$. Each column represents mean \pm standard error of the mean. $* p<0.05$, $* * p<0.01$ vs. control mice. n. s., not significant; Control, control group fed AIN-93M; HFD, HFD group fed HFD-60.

Yeast Co., Tokyo, Japan) or an HFD group fed HFD-60 (Oriental Yeast Co.) for 8 weeks. The composition of AIN93M and HFD-60 is shown in Supplementary Table S1.

All experiments were carried out under the control of animal research committee in accordance with the Guidelines on Animal Experiments in Osaka City University Graduate School of Medicine, the Japanese 

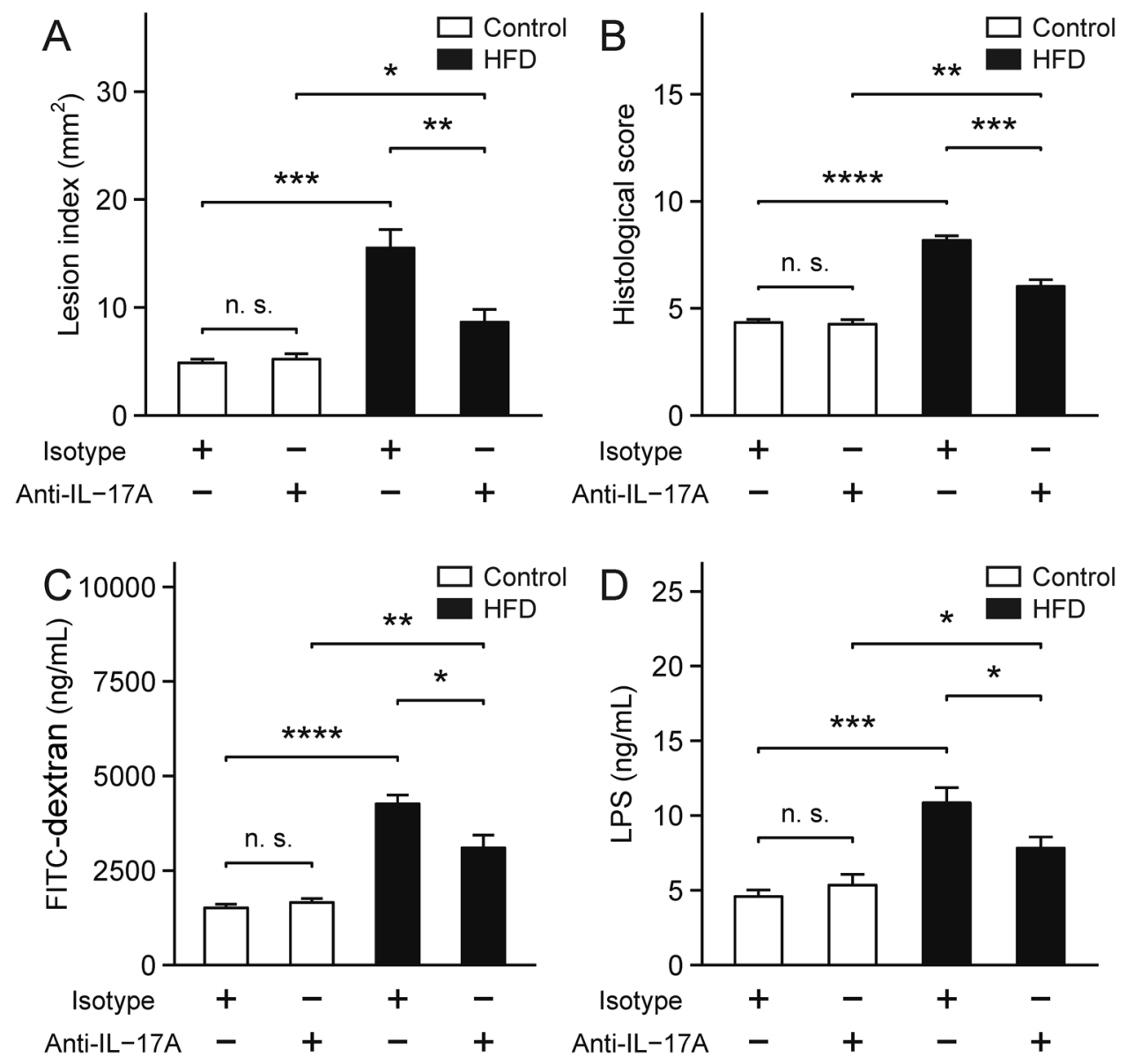

Figure 5. Effects of neutralization of IL-17A. (A) Lesion index of indomethacin-induced small intestinal damage. $n=7-10$. (B) Histological evaluation of indomethacin-induced small intestinal damage. $n=5$. $($ C) Intestinal permeability was examined by measuring serum FITC-dextran levels. $n=8-10$. (D) Serum LPS levels were analyzed using the murine LPS ELISA kit. $\mathrm{n}=9-10$. Each column represents mean \pm standard error of the mean. $* p<0.05, * * p<0.01, * * * p<0.001$, **** $p<0.0001$; n. s., not significant; Isotype, mice treated with isotype control; Anti-IL-17A, mice treated with anti-IL-17A antibody; Control, control group fed AIN-93M; HFD, HFD group fed HFD-60.

Government Animal Protection and Management Law (No. 105), and the Japanese Government Notification on Feeding and Safekeeping of Animals (No. 6). All experimental procedures were approved by the Animal Care Committee of Osaka City University Graduate School of Medicine (Approval number 16014). All surgeries were performed under isoflurane, with maximal efforts taken to minimize suffering.

Induction of experimental small intestinal damage. Non-fasted mice were administered indomethacin (12 mg/kg BW; Sigma-Aldrich, St Louis, MO, USA) in 0.5\% carboxymethylcellulose solution by gavage and were sacrificed $24 \mathrm{~h}$ later. Small intestinal damage was assessed using 1\% Evans blue by intravenous injection $30 \mathrm{~min}$ before sacrifice. The small intestine was opened along the antimesenteric attachment and examined for damage under a dissecting microscope with square grids $(\times 10)$. The area $\left(\mathrm{mm}^{2}\right)$ of macroscopically visible lesion was measured, summed per small intestine, and used as the lesion index. Prior to indomethacin treatment, tissue samples with luminal contents were collected $(4.5 \mathrm{~cm}$ in length each) from lower ileum section at a position approximately $2 \mathrm{~cm}$ proximal to the terminal ileum. Blood samples were obtained by cardiac puncture.

Histological examination of small intestinal damage. Tissue samples were fixed in $0.1 \mathrm{M}$ phosphate buffer (pH 7.4) containing 4\% paraformaldehyde and embedded in OCT compound (Miles, Elkhart, IN, USA). Serial 5- $\mu \mathrm{m}$-thick cryostat sections were mounted on silanized slides (Dako, Tokyo, Japan) and stained with hematoxylin and eosin. For each mouse, at least ten random villi at the damaged areas were scored in a masked fashion by 2 investigators independently. For quantitative evaluation, a modified histological scoring system was used $^{18}$. Total histology scores ranged from 0 to 13 and were evaluated for 6 categories with sub-scores as follows: epithelium ( 0 , normal; 1 , flattened; 2 , loss of epithelial continuity; 3 , severe denudation), villus shape ( 0 , normal; 1 , short and rounded; 2 , extremely short and thick), villus tip ( 0 , normal; 1 , damaged; 2 , severely damaged), stroma ( 0 , normal; 1 , slightly retracted; 2 , severely retracted), inflammation ( 0 , no infiltration; 1 , mild infiltration; 2 , severe infiltration), and crypt status ( 0 , normal; 1 , mild crypt loss; 2 , severe crypt loss). 
A
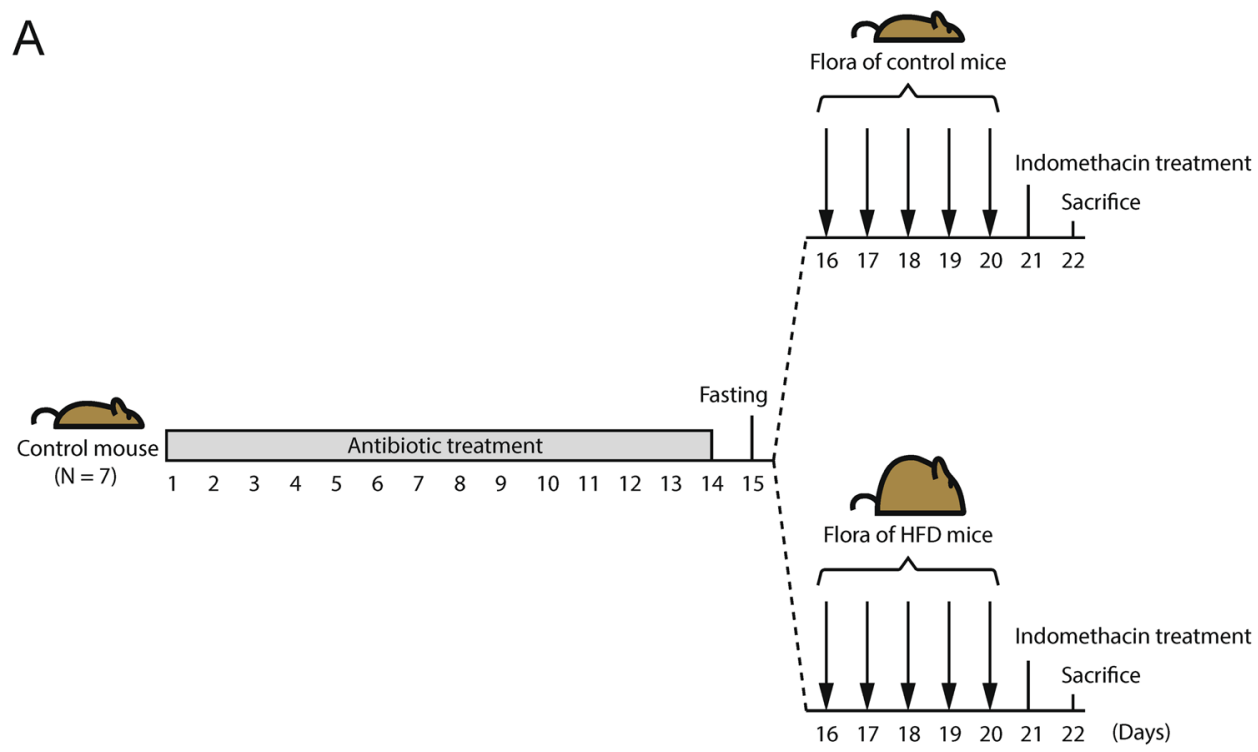

B

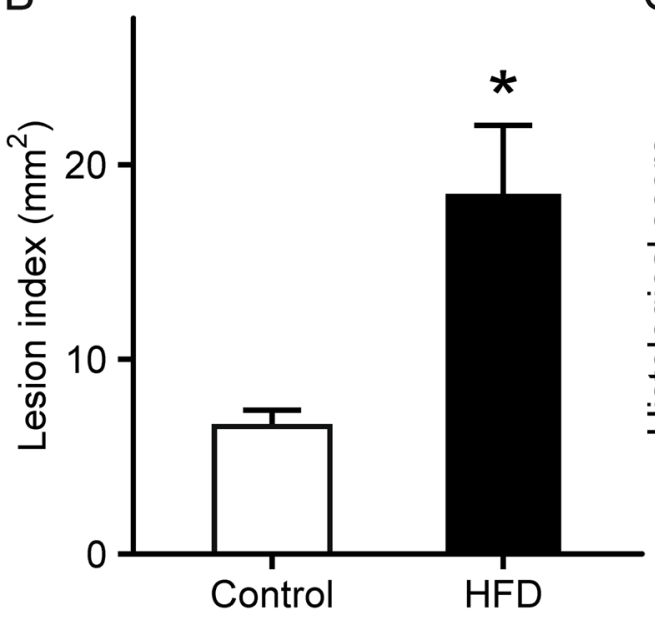

C

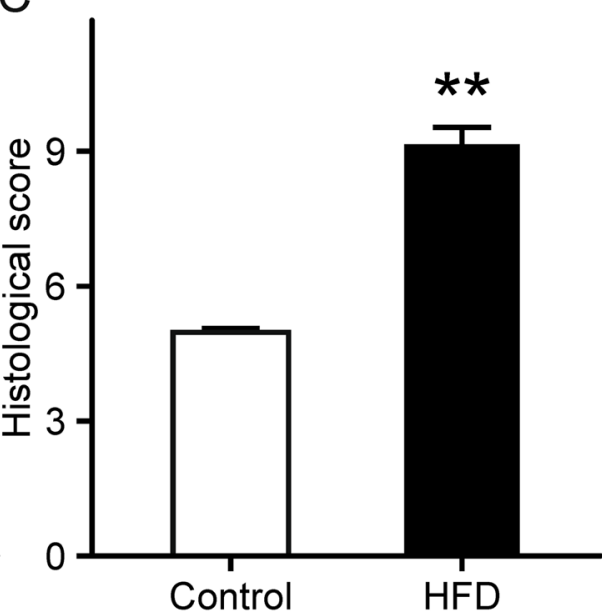

Figure 6. Effects of small intestinal microbiota transplantation. (A) Schematic timeline of small intestinal microbiota transplantation. (B) Lesion index of indomethacin-induced small intestinal damage after microbial transplantation from donor mice fed HFD-60 or AIN-93M. $n=7-8$. (C) Histological evaluation of indomethacin-induced small intestinal damage after microbial transplantation from donor mice fed HFD-60 or AIN-93M. $\mathrm{n}=4$. Each column represents mean \pm standard error of the mean. $* p<0.05, * * p<0.01$ vs. control mice. Control, mice that received microbial transplantation from donor mice fed AIN-93M; HFD, mice that received microbial transplantation from donor mice fed HFD-60.

DNA extraction and 16S rRNA gene analysis. Small intestinal luminal contents were collected immediately after sacrifice, using a feces collection kit (Takara Bio, Shiga, Japan). Genomic DNA was then isolated using the NucleoSpin Microbial DNA Kit (MACHEREY-NAGEL, Düren, Germany). Approximately $500 \mu$ l of stored small intestinal samples were placed in a microcentrifuge tube containing $100 \mu$ l elution buffer. The mixture was placed into the NucleoSpin Beads Tube with proteinase K, which was subjected to mechanical beads-beating for $12 \mathrm{~min}$ at $30 \mathrm{~Hz}$ in the TissueLyzer LT. DNA extraction was subsequently performed as per manufacturer's instructions. DNA samples were purified using the Agencourt AMPure XP (Beckman Coulter, Brea, CA).

V3-4 amplicon sequences of 16S rRNA genes were prepared as described previously ${ }^{34}$. Briefly, 16S rRNA gene fragments including V3 and V4 regions were amplified by PCR (forward primer: ACACGAC GCTCTTCCGATCTCCTACGGGNGGCWGCAG, reverse primer: GACGTGTGCTCTTCCGATC TGACTACHVGGGTATCTAATCC) for 20 cycles, and PCR products were purified with Agencourt AMpure beads (Beckman Coulter). Next, index sequences for sequencing were added by running a second PCR with NEBNext Multiplex Oligos for Illumina (Dual Index Primers Set1, New England Biolabs) for 8 cycles, and the products were purified with Agencourt AMpure beads. For each sample, an equal amount of each DNA amplicon library was mixed and sequenced on the MiSeq instrument (Illumina) using a MiSeq v3 Reagent kit with 20\% PhiX (Illumina). 
FITC-Dextran permeability assay. Intestinal permeability was measured as previously described with some modifications ${ }^{10}$. Mice were fasted for $16 \mathrm{~h}$ and FITC-dextran was administered by gavage $(500 \mathrm{mg} / \mathrm{kg} \mathrm{BW}$, $125 \mathrm{mg} / \mathrm{ml}$; Sigma-Aldrich). After $4 \mathrm{~h}$ of administration, $120 \mu \mathrm{l}$ of blood was collected from the heart, stored on ice in the dark, and centrifuged $(15,000 \mathrm{rpm})$ at $4{ }^{\circ} \mathrm{C}$ for $10 \mathrm{~min}$. Serum was diluted in an equal volume of PBS (pH 7.4) and analyzed for FITC-dextran concentration with a fluorescence spectrophotometer (Varioskan LUX; Thermo Scientific, Waltham, MA, USA) at an excitation wavelength of $485 \mathrm{~nm}$ and an emission wavelength of $535 \mathrm{~nm}$.

Blood sampling and LPS assay. Blood samples were obtained by cardiac puncture and stored on ice for $30 \mathrm{~min}$ before centrifugation $(15,000 \mathrm{rpm})$ at $4{ }^{\circ} \mathrm{C}$ for $10 \mathrm{~min}$. Serum portions were isolated, and LPS levels were measured with the murine LPS ELISA kit (Cusabio, Wuhan, China).

Real-time quantitative RT-PCR. Total RNA was extracted from small intestinal tissues with the ISOGEN kit (Nippon Gene Co., Ltd., Tokyo, Japan). Real-time quantitative RT-PCR analyses were performed as previously described ${ }^{35}$. The sequences of PCR primers and TaqMan probes of ZO-1, MCP-1, IL-1 $\beta$, TNF- $\alpha$, and TLR 4 are shown in Supplementary Table S2. TaqMan Gene Expression Assays (Life Technologies Corp., Rockville, MD, USA) were used for measuring occludin, IL-17A, and TLR9. Glyceraldehyde-3-phosphate dehydrogenase (GAPDH) was used as an internal control, and mRNA levels were expressed as ratios relative to the mean of control mouse data.

Western blotting. Small intestinal tissues were homogenized and lysed on ice in buffer containing $0.5 \%$ NP-40, $40 \mathrm{mM}$ Tris- $\mathrm{HCl}$ (pH 8.0), $120 \mathrm{mM} \mathrm{NaCl}, 1 \mathrm{mM}$ PMSF, and $10 \mu \mathrm{g} / \mathrm{ml}$ leupeptin. Proteins in lysates were measured with a modified bicinchoninic acid method. Proteins were denatured with SDS sample buffer, subjected to $10 \%$ SDS-polyacrylamide gel electrophoresis, and transferred to PVDF membrane. Membranes were blocked with TBS buffer $(10 \mathrm{mM}$ Tris- $\mathrm{HCl}, \mathrm{pH} 7.5,100 \mathrm{mM} \mathrm{NaCl}$, and $0.1 \%$ Tween-20) containing $5 \%$ BSA and were incubated overnight with rabbit polyclonal antibodies against ZO-1 (catalog no. 61-7300, Invitrogen, Carlsbad, CA, USA; diluted 1:1000), or rabbit monoclonal antibodies against occludin (catalog no. ab168986, Abcam, Cambridge, UK; diluted 1:1000). Antigen-antibody complexes were detected with anti-rabbit IgG-HRP (diluted 1:1000) using enhanced chemiluminescence in accordance with the manufacturer's instructions (Amersham, Arlington Heights, IL, USA). Bands were quantified using laser-scanning densitometry and the expression level of each protein was normalized against that of $\beta$-actin. Full-length Western blots of ZO- 1 and occludin are shown in Supplementary Fig. S3.

Neutralization of IL-17A. After 8 weeks on control diet or HFD, mice were subjected to intraperitoneal injection of anti-IL-17A antibody (4 mg/kg; clone 17F3, mouse IgG1, BioXCell, West Lebanon, NH, USA) or mouse IgG1 isotype control (4 mg/kg; clone MOPC-21, mouse IgG1, BioXCell) once daily for 7 days. Two days after the final administration of antibodies, mice were administered indomethacin $(12 \mathrm{mg} / \mathrm{kg}$; Sigma-Aldrich $)$ in a $0.5 \%$ carboxymethylcellulose solution by gavage and sacrificed $24 \mathrm{~h}$ later.

Small intestinal microbiota transplantation. After 8 weeks on control diet, recipient mice were given drinking water containing ampicillin $(1 \mathrm{~g} / \mathrm{l})$, metronidazole $(1 \mathrm{~g} / \mathrm{l})$, neomycin $(1 \mathrm{~g} / \mathrm{l})$, and vancomycin $(0.5 \mathrm{~g} / \mathrm{l})$ for 2 weeks. Small intestinal luminal contents from donor mice were collected, dissolved in $10 \mathrm{~g} / \mathrm{L}$ sterile PBS, centrifuged at $1,500 \mathrm{rpm}$ for $5 \mathrm{~min}$. Supernatants were administered to the recipient mice by gavage $(0.5 \mathrm{ml} / \mathrm{mouse})$ for 5 days. After the final day of transplantation, mice were administered indomethacin ( $12 \mathrm{mg} / \mathrm{kg} \mathrm{BW}$; Sigma-Aldrich) in a $0.5 \%$ carboxymethylcellulose solution by gavage and sacrificed $24 \mathrm{~h}$ later.

Statistical analysis. Values are expressed as mean \pm standard error of the mean (SEM) in animal experiments. Comparisons between 2 groups were performed using a two-sided Student's $t$-test. ANOVA was used to compare differences among multiple groups, and post-hoc analysis was performed by Tukey's multiple comparisons test. $P$-values $<0.05$ indicate a statistical significance.

Received: 26 July 2019; Accepted: 25 October 2019;

Published online: 14 November 2019

\section{References}

1. Graham, D. Y., Opekun, A. R., Willingham, F. F. \& Qureshi, W. A. Visible small-intestinal mucosal injury in chronic NSAID users. Clin Gastroenterol Hepatol 3, 55-59, https://doi.org/10.1016/S1542-3565(04)00603-2 (2005).

2. Sugimori, S. et al. Evaluation of small bowel injury in patients with rheumatoid arthritis by capsule endoscopy: effects of antirheumatoid arthritis drugs. Digestion 78, 208-213, https://doi.org/10.1159/000190403 (2008).

3. Watanabe, T. et al. Risk factors for severe nonsteroidal anti-inflammatory drug-induced small intestinal damage. Dig Liver Dis 45, 390-395, https://doi.org/10.1016/j.dld.2012.12.005 (2013).

4. Wallace, J. L. et al. Proton pump inhibitors exacerbate NSAID-induced small intestinal injury by inducing dysbiosis. Gastroenterology 141, 1314-1322, 1322 e1311-1315, https://doi.org/10.1053/j.gastro.2011.06.075 (2011).

5. Tamboli, C. P., Neut, C., Desreumaux, P. \& Colombel, J. F. Dysbiosis in inflammatory bowel disease. Gut 53, 1-4, https://doi. org/10.1136/gut.53.1.1 (2004).

6. Henao-Mejia, J. et al. Inflammasome-mediated dysbiosis regulates progression of NAFLD and obesity. Nature 482, 179-185, https:// doi.org/10.1038/nature10809 (2012).

7. Sobhani, I. et al. Microbial dysbiosis in colorectal cancer (CRC) patients. PLoS One 6, e16393, https://doi.org/10.1371/journal. pone.0016393 (2011).

8. Murphy, E. A., Velazquez, K. T. \& Herbert, K. M. Influence of high-fat diet on gut microbiota: a driving force for chronic disease risk. Curr Opin Clin Nutr Metab Care 18, 515-520, https://doi.org/10.1097/MCO.0000000000000209 (2015). 
9. Hamilton, M. K., Boudry, G., Lemay, D. G. \& Raybould, H. E. Changes in intestinal barrier function and gut microbiota in high-fat diet-fed rats are dynamic and region dependent. Am J Physiol Gastrointest Liver Physiol 308, G840-851, https://doi.org/10.1152/ ajpgi.00029.2015 (2015)

10. Cani, P. D. et al. Changes in gut microbiota control metabolic endotoxemia-induced inflammation in high-fat diet-induced obesity and diabetes in mice. Diabetes 57, 1470-1481, https://doi.org/10.2337/db07-1403 (2008).

11. Cani, P. D. et al. Changes in gut microbiota control inflammation in obese mice through a mechanism involving GLP-2-driven improvement of gut permeability. Gut 58, 1091-1103, https://doi.org/10.1136/gut.2008.165886 (2009).

12. Wang, Z. et al. The role of bifidobacteria in gut barrier function after thermal injury in rats. J Trauma 61, 650-657, https://doi. org/10.1097/01.ta.0000196574.70614.27 (2006).

13. Cani, P. D. et al. Metabolic endotoxemia initiates obesity and insulin resistance. Diabetes 56, 1761-1772, https://doi.org/10.2337/ db06-1491 (2007).

14. Araujo, J. R., Tomas, J., Brenner, C. \& Sansonetti, P. J. Impact of high-fat diet on the intestinal microbiota and small intestinal physiology before and after the onset of obesity. Biochimie 141, 97-106, https://doi.org/10.1016/j.biochi.2017.05.019 (2017).

15. Bjarnason, I., Fehilly, B., Smethurst, P., Menzies, I. S. \& Levi, A. J. Importance of local versus systemic effects of non-steroidal antiinflammatory drugs in increasing small intestinal permeability in man. Gut 32, 275-277, https://doi.org/10.1136/gut.32.3.275 (1991).

16. Watanabe, T. et al. Non-steroidal anti-inflammatory drug-induced small intestinal damage is Toll-like receptor 4 dependent. Gut 57, 181-187, https://doi.org/10.1136/gut.2007.125963 (2008).

17. Higashimori, A. et al. Mechanisms of NLRP3 inflammasome activation and its role in NSAID-induced enteropathy. Mucosal Immunol 9, 659-668, https://doi.org/10.1038/mi.2015.89 (2016).

18. Nadatani, Y. et al. High mobility group box 1 promotes small intestinal damage induced by nonsteroidal anti-inflammatory drugs through Toll-like receptor 4. Am J Pathol 181, 98-110, https://doi.org/10.1016/j.ajpath.2012.03.039 (2012).

19. Wu, H. M. et al. CpG-ODN promotes phagocytosis and autophagy through JNK/P38 signal pathway in Staphylococcus aureusstimulated macrophage. Life Sci 161, 51-59, https://doi.org/10.1016/j.lfs.2016.07.016 (2016).

20. Hemmi, H. et al. A Toll-like receptor recognizes bacterial DNA. Nature 408, 740-745, https://doi.org/10.1038/35047123 (2000).

21. Sardi, C. et al. Three months of Western diet induces small intestinal mucosa alteration in TLR KO mice. Microsc Res Tech $\mathbf{8 0}$, 563-569, https://doi.org/10.1002/jemt.22831 (2017).

22. Akdis, M., Palomares, O., van de Veen, W., van Splunter, M. \& Akdis, C. A. TH17 and TH22 cells: a confusion of antimicrobial response with tissue inflammation versus protection. J Allergy Clin Immunol 129, 1438-1449, https://doi.org/10.1016/j.jaci.2012.05.003 (2012). quiz1450-1431.

23. Nakamizo, S. et al. High fat diet exacerbates murine psoriatic dermatitis by increasing the number of IL-17-producing gammadelta T cells. Sci Rep 7, 14076, https://doi.org/10.1038/s41598-017-14292-1 (2017).

24. Bibi, S., Kang, Y., Du, M. \& Zhu, M. J. Maternal high-fat diet consumption enhances offspring susceptibility to DSS-induced colitis in mice. Obesity (Silver Spring) 25, 901-908, https://doi.org/10.1002/oby.21816 (2017).

25. Shen, T. et al. Interleukin-17A exacerbates high-fat diet-induced hepatic steatosis by inhibiting fatty acid beta-oxidation. Biochim Biophys Acta Mol Basis Dis 1863, 1510-1518, https://doi.org/10.1016/j.bbadis.2017.01.027 (2017).

26. Babu, S. T. et al. Maternal high-fat diet results in microbiota-dependent expansion of ILC3s in mice offspring. JCI Insight 3, https:// doi.org/10.1172/jci.insight.99223 (2018).

27. Ivanov, II \& Littman, D. R. Segmented filamentous bacteria take the stage. Mucosal Immunol 3, 209-212, https://doi.org/10.1038/ mi.2010.3 (2010).

28. Meng, J. et al. Opioid Exacerbation of Gram-positive sepsis, induced by Gut Microbial Modulation, is Rescued by IL-17A Neutralization. Sci Rep 5, 10918, https://doi.org/10.1038/srep10918 (2015).

29. Uejima, M., Kinouchi, T., Kataoka, K., Hiraoka, I. \& Ohnishi, Y. Role of intestinal bacteria in ileal ulcer formation in rats treated with a nonsteroidal antiinflammatory drug. Microbiol Immunol 40, 553-560, https://doi.org/10.1111/j.1348-0421.1996.tb01108.x (1996).

30. Konaka, A. et al. Roles of enterobacteria, nitric oxide and neutrophil in pathogenesis of indomethacin-induced small intestinal lesions in rats. Pharmacol Res 40, 517-524, https://doi.org/10.1006/phrs.1999.0550 (1999).

31. Hagiwara, M., Kataoka, K., Arimochi, H., Kuwahara, T. \& Ohnishi, Y. Role of unbalanced growth of gram-negative bacteria in ileal ulcer formation in rats treated with a nonsteroidal anti-inflammatory drug. J Med Invest 51, 43-51, https://doi.org/10.2152/jmi.51.43 (2004).

32. Otani, K. et al. Microbiota Plays a Key Role in Non-Steroidal Anti-Inflammatory Drug-Induced Small Intestinal Damage. Digestion 95, 22-28, https://doi.org/10.1159/000452356 (2017).

33. van Nood, E. et al. Duodenal infusion of donor feces for recurrent Clostridium difficile. N Engl J Med 368, 407-415, https://doi. org/10.1056/NEJMoa1205037 (2013).

34. Usui, Y. et al. Effects of long-term intake of a yogurt fermented with Lactobacillus delbrueckii subsp. bulgaricus 2038 and Streptococcus thermophilus 1131 on mice. Int Immunol 30, 319-331, https://doi.org/10.1093/intimm/dxy035 (2018).

35. Itani, S. et al. NLRP3 inflammasome has a protective effect against oxazolone-induced colitis: a possible role in ulcerative colitis. Sci Rep 6, 39075, https://doi.org/10.1038/srep39075 (2016).

\section{Acknowledgements}

We appreciate Emi Suzuki-Yoshioka for her technical assistance.

\section{Author contributions}

N.S. performed the experiments, analyzed data, and drafted the paper; K.O. provided technical support and revised the paper; T.W. designed, supervised this study, and revised the paper; N.S. and S.S. evaluated the histology scores; G.N., K.F. and S.U. performed the molecular analysis of small intestinal microbiome; Y.N., S.H., F.T., N.K., K.T., T.T. and Y.F. reviewed the manuscript and provided valid inputs on the study.

\section{Competing interests}

The authors declare no competing interests.

\section{Additional information}

Supplementary information is available for this paper at https://doi.org/10.1038/s41598-019-52980-2.

Correspondence and requests for materials should be addressed to T.W.

Reprints and permissions information is available at www.nature.com/reprints.

Publisher's note Springer Nature remains neutral with regard to jurisdictional claims in published maps and institutional affiliations. 
(c) (i) Open Access This article is licensed under a Creative Commons Attribution 4.0 International License, which permits use, sharing, adaptation, distribution and reproduction in any medium or format, as long as you give appropriate credit to the original author(s) and the source, provide a link to the Creative Commons license, and indicate if changes were made. The images or other third party material in this article are included in the article's Creative Commons license, unless indicated otherwise in a credit line to the material. If material is not included in the article's Creative Commons license and your intended use is not permitted by statutory regulation or exceeds the permitted use, you will need to obtain permission directly from the copyright holder. To view a copy of this license, visit http://creativecommons.org/licenses/by/4.0/.

(C) The Author(s) 2019 